\title{
Achieving Good Angular Resolution in 3D Arc Diagrams
}

\author{
Michael T. Goodrich and Paweł Pszona \\ Dept. of Computer Science \\ University of California, Irvine
}

\begin{abstract}
We study a three-dimensional analogue to the well-known graph visualization approach known as arc diagrams. We provide several algorithms that achieve good angular resolution for 3D arc diagrams, even for cases when the arcs must project to a given 2D straight-line drawing of the input graph. Our methods make use of various graph coloring algorithms, including an algorithm for a new coloring problem, which we call localized edge coloring.
\end{abstract}

\section{Introduction}

An arc diagram is a two-dimensional graph drawing where the vertices of a graph, $G$, are placed on a one-dimensional curve (typically a straight line) and the edges of $G$ are drawn as circular arcs that may go outside that curve (e.g.,

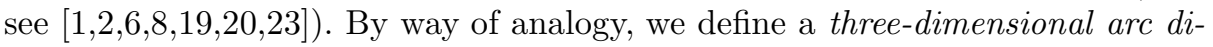
agram to be a drawing where the vertices of a graph, $G$, are placed on a twodimensional surface (such as a sphere or plane) and the edges of $G$ are drawn as circular arcs that may go outside that surface. (See Fig. 1.) This 3D drawing paradigm is used, for example, to draw geographic networks or flight networks (e.g., see [3]).

In this paper, we are interested in the angular resolution of 3D arc diagrams, that is, the smallest angle determined by the tangents at a vertex, $v$, to two

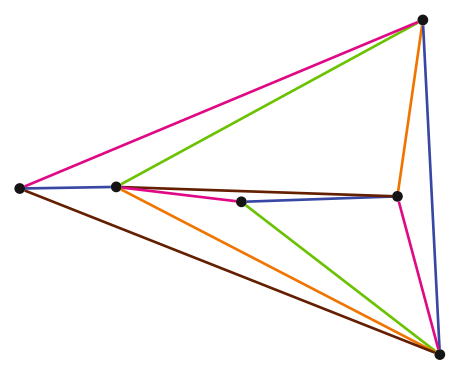

(a)

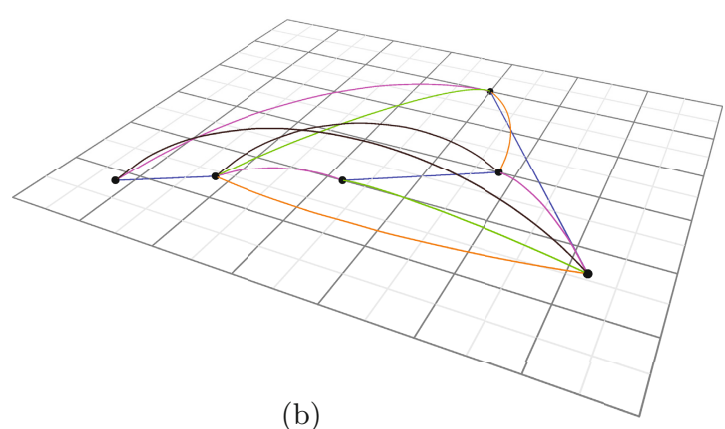

(b)

Fig. 1. A graph rendered (a) as a straight-line drawing and (b) as a 3D arc diagram 
arcs incident to $v$ in such a drawing. Specifically, we provide algorithms for achieving good angular resolution in 3D arc diagrams where the (base) surface that contains the vertices for the graph, $G$, is a sphere or a plane. Moreover, for the $3 \mathrm{D}$ arc diagrams that we consider in this paper, we assume that all the edges of $G$ are drawn to protrude out of only one side of the base surface.

\subsection{Previous Related Results}

The term "arc diagram" was defined in 2002 by Wattenberg [23], but the drawing paradigm actually can be traced back to the 1960's, including work by Saaty [20] and Nicholson [19]. Also, earlier work by Brandes [2] explores symmetry in arc diagrams, earlier work by Cimikowski and Shope [6] explores heuristics for minimizing the number of arc crossings, and earlier work by Djidjev and Vrt'o [8] explores lower bounds for the crossing numbers of such drawings. Most recently, Angelini et al. [1] show that there is a universal set of $O(n)$ points on a parabola that allows any planar graph to be drawn as a planar arc diagram.

In terms of previous work on arc diagrams for optimizing the angular resolution of such drawings, Duncan et al. [11] give a complete characterization of which regular graphs can be drawn as arc diagrams with vertices placed on a circle and perfect angular resolution, using a drawing style inspired by the artist, Mark Lombardi, where edges are drawn using circular arcs so as to achieve good angular resolution. With respect to a lower bound for this drawing style, Cheng et al. 5] give a planar graph with bounded degree, $d$, that requires exponential area if it is drawn as a plane graph with circular-arc edges and angular resolution $\Omega(1 / d)$. Even so, it is possible to draw any planar graph as a plane graph with poly-line or poly-circular edges to achieve polynomial area and $\Omega(1 / d)$ angular resolution, based on results by a number of authors (e.g., see Brandes et al. 4], Cheng et al. [5], Duncan et al. 911, Garg and Tamassia [15, Goodrich and Wagner [17, and Gutwenger and Mutzel [18).

In addition, several researchers have investigated how to achieve good angular resolution for various straight-line drawings of graphs. Duncan et al. [10] show that one can draw an ordered tree of degree $d$ as a straight-line planar drawing with angular resolution $\Omega(1 / d)$. Formann et al. 14] show that any graph of degree $d$ has a straight-line drawing with polynomial area and angular resolution $\Omega\left(1 / d^{2}\right)$, and this can be improved to be $\Omega(1 / d)$ for planar graphs, albeit with a drawing that may not be planar.

We are not familiar with any previous work on achieving good angular resolution for 3D arc diagrams, but there is previous related work on other types of 3D drawings [7. For instance, Brandes et al. 3] show that one can achieve $\Omega(1 / d)$ angular resolution for 3D geometric network drawings, but their edges are curvilinear splines, rather than simple circular arcs. Garg et al. [16] study $3 \mathrm{D}$ straight-line drawings so as to satisfy various resolution criteria, but they do not constrain vertices to belong to a 2D surface. In addition, Eppstein et al. [12 provide an algorithm for achieving optimal angular resolution in 3D drawings of low-degree graphs using poly-line edges. 


\subsection{Our Results}

In this paper, we give several algorithms for achieving good angular resolution for 3D arc diagrams. In particular, we show the following for a graph, $G$, with maximum degree, $d$ :

- We can draw $G$ as a 3D arc diagram with an angular resolution of $\Omega(1 / d)$ $\left(\Omega\left(1 / d^{1 / 2}\right)\right.$ if $G$ is planar) using straight-line segments and vertices placed on a sphere.

- We can draw $G$ as a 3D arc diagram with an angular resolution of $\Omega(1 / d)$ using circular arcs that project perpendicularly to a given straight-line drawing for $G$ in a base plane, no matter how poor the angular resolution of that projected drawing.

- If a straight-line 2D drawing of $G$ already has an angular resolution of $\Omega(1 / d)$ in a base plane, $\mathcal{P}$, then we can draw $G$ as a $3 \mathrm{D}$ arc diagram with an angular resolution of $\Omega\left(1 / d^{1 / 2}\right)$ using circular arcs that project perpendicularly to the given drawing of $G$ in $\mathcal{P}$.

- Given any 2D straight-line drawing of $G$ in a base plane, $\mathcal{P}$, we can draw $G$ as a $3 \mathrm{D}$ arc diagram with an angular resolution of $\Omega\left(1 / d^{1 / 2}\right)$ using circular arcs that project to the edges of the drawing of $G$ in $\mathcal{P}$, with each arc possibly using a different projection direction.

Our algorithms make use of various graph coloring methods, including an algorithm for a new coloring problem, which we call localized edge coloring.

Note that $O\left(1 / d^{1 / 2}\right)$ is an upper bound on the resolution of a 3D arc drawing of $G$, as maximizing the smallest angle between two edges around a vertex, $v$, is equivalent to maximizing smallest distance between intersections of a unit sphere centered at $v$, and lines tangent to edges incident to $v$, which is known as the Tammes problem [21]. The $O\left(1 / d^{1 / 2}\right)$ upper bound is due to Fejes Tóth [13].

\section{Preliminaries}

In this section, we provide formal definitions of two notions of 3D arc diagrams.

We extend the notion of arc diagrams and define $3 D$ arc diagram drawings of a graph, $G$, to be 3D drawings that meet the following criteria:

(1) nodes (vertices) are placed on a single (base) sphere or plane

(2) each edge, $e$, is drawn as a circular arc, i.e., a contiguous subset of a circle

(3) all edges lie entirely on one side of the base sphere or plane.

In addition, if the base surface is a plane, $\mathcal{P}_{1}$, then each circular edge, $e$, which belongs to a plane, $\mathcal{P}_{2}$, forms the same angle, $\alpha_{e} \leq \pi / 2$, in $\mathcal{P}_{2}$, at its two endpoints. Moreover, in this case, each edge projects (perpendicularly) to a straight line segment in $\mathcal{P}_{1}$. An example of such an arc is shown in Fig. 2ak. 


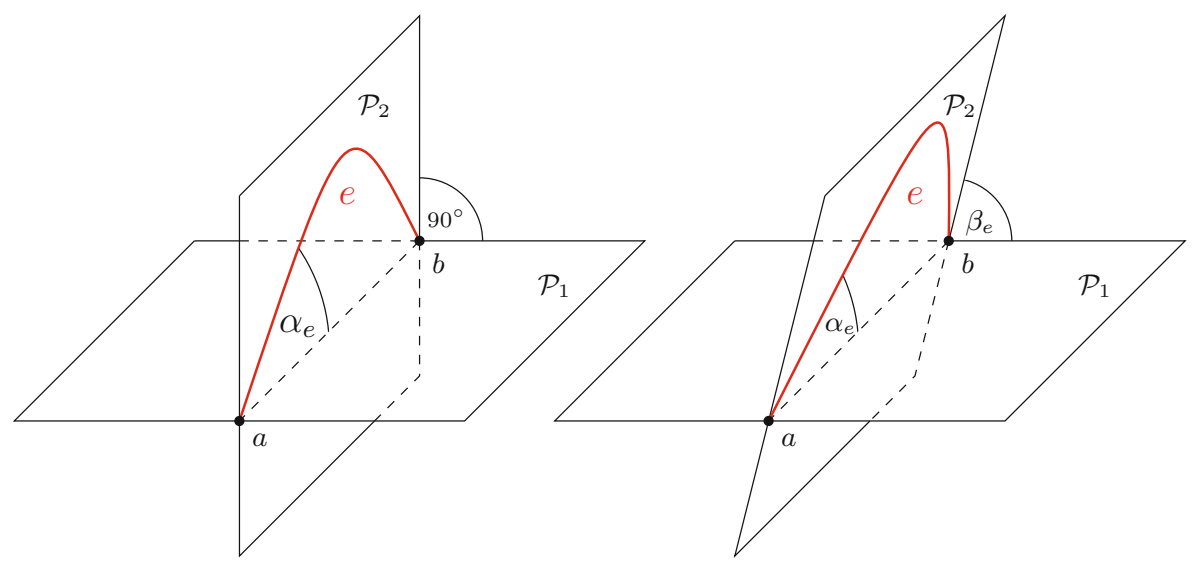

(a)

(b)

Fig. 2. Edge $e=(a, b)$ drawn as (a) circular arc with angle $\alpha_{e}$; (b) slanted circular arc with angles $\left(\alpha_{e}, \beta_{e}\right)$.

For $3 \mathrm{D}$ arc diagrams restricted to use a base plane, $\mathcal{P}_{1}$ (rather than a sphere), by modifying the second condition, we obtain a definition of slanted $3 D$ arc diagram drawings.

$\left(2^{\prime}\right)$ each edge $e$ is a circular arc that lies on a plane, $\mathcal{P}_{2}$, that contains both endpoints of $e$ and forms an angle, $\beta_{e}<\pi / 2$, with the base plane, $\mathcal{P}_{1}$; the edge, $e$, forms the same angle, $\alpha_{e} \leq \pi / 2$, in $\mathcal{P}_{2}$, at its two endpoints.

Note that in this case each circular edge, $e$, joining vertices $a$ and $b$, in a slanted $3 \mathrm{D}$ arc diagram, projects to a straight line segment, $L=a b$, in the base plane, $\mathcal{P}_{1}$, using a direction perpendicular to $L$ in $\mathcal{P}_{2}$. Still, a perpendicular projection of the drawing onto the base plane, $\mathcal{P}_{1}$, is not necessarily a straight-line drawing of $G$ and may not even be planar. For an example, see Fig. 2b.

\section{Localized Edge Coloring}

Recall that a vertex coloring of a graph is an assignment of colors to vertices so that every vertex is given a color different from those of its adjacent vertices, and an edge coloring is an assignment of colors to a graph's edges so that every edge is given a color different from its incident edges. A well-known greedy algorithm can color any graph with maximum degree, $d$, using $d+1$ colors, and Vizing's theorem 22] states that edges of an undirected graph $G$ can similarly be colored with $d+1$ colors, as well.

Assuming we are given an undirected graph $G$ together with its combinatorial embedding on a plane (i.e., the order of edges around each vertex, which is also known as a rotation system), we introduce a localized notion of an edge coloring, which will be useful for some of our results regarding 3D arc diagrams. Given an 
even integer parameter, $L$, we define an $L$-localized edge coloring to be an edge coloring that satisfies the following condition:

Suppose an edge $e=(u, v)$ has color $c$, and let $\left(l_{1}, l_{2}, \ldots, l_{i}=e, \ldots l_{k}\right)$ be a clockwise ordering of edges incident to $u$. Then none of the edges $l_{i-L / 2}, l_{i-L / 2+1}, \ldots, l_{i-1}, l_{i+1}, \ldots, l_{i+L / 2}$, that is, the $L / 2$ edges before $e$ and $L / 2$ edges after $e$ in the ordering, has color $c$. (Note that, by symmetry, the same goes for edges around $v$.)

Thus, a valid $d$-localized edge coloring is also a valid classical edge coloring. We call the set, $\left\{l_{i-L / 2}, l_{i-L / 2+1}, \ldots, l_{i-1}, l_{i+1}, \ldots, l_{i+L / 2}\right\}$, the $L$-neighborhood of $e$ around $u$.

As with the greedy approach to vertex coloring, an $L$-localized edge coloring can be found by a simple greedy algorithm that incrementally assigns colors to edges, one at a time. Each edge $e=(u, v)$ is colored with color $c$ that does not appear in both $L$-neighborhoods of $e$ (around $u$ and around $v$ ). Using reasonable data structures, this greedy algorithm can be implemented to run in $O(m L)$ time, for a graph with $m$ edges, and combining it with Vizing's theorem [22], allows us to find an edge coloring that uses at most $\min \{d, 2 L\}+1$ colors.

\section{Improving Resolution via Edge Coloring}

As mentioned above, we define the angle between two incident arcs in the 3D arc diagram to be the angle between lines tangent to the arcs at their common endpoint. In order to reason about angles in $3 \mathrm{D}$, the following lemma will prove useful.

Lemma 1. Consider two segments $l_{1}, l_{2}$ that share a common endpoint that lies on a plane $\mathcal{P}$ (see Fig. 3). If both $l_{1}$ and $l_{2}$ form angle $\beta \leq \pi / 4$ with their projections onto $\mathcal{P}$, and projections of $l_{1}$ and $l_{2}$ onto $\mathcal{P}$ form angle $\alpha$, then $\delta$, the angle between $l_{1}$ and $l_{2}$, is at least $\alpha / 2$.

Proof. Assume w.l.o.g. that $\left|l_{1}\right|=\left|l_{2}\right|=1$. The distance $d$ between endpoints of $l_{1}$ and $l_{2}$ is the same as the distance between endpoints of projections of $l_{1}$ and $l_{2}$ onto $\mathcal{P}$ (because both $l_{1}$ and $l_{2}$ form angle $\beta$ with $\mathcal{P}$ ). Lengths of the projections are $\cos \beta$, and by the law of cosines,

$$
d^{2}=\cos ^{2} \beta+\cos ^{2} \beta-2 \cos \beta \cos \beta \cos \alpha=2 \cos ^{2} \beta(1-\cos \alpha) .
$$

On the other hand, again by the law of cosines,

$$
d^{2}=\left|l_{1}\right|^{2}+\left|l_{2}\right|^{2}-2\left|l_{1}\right|\left|l_{2}\right| \cos \delta=2(1-\cos \delta) .
$$

Comparing the two yields

$$
2 \cos ^{2} \beta(1-\cos \alpha)=2(1-\cos \delta),
$$

which leads to

$$
\cos \delta=1-\cos ^{2} \beta(1-\cos \alpha)
$$




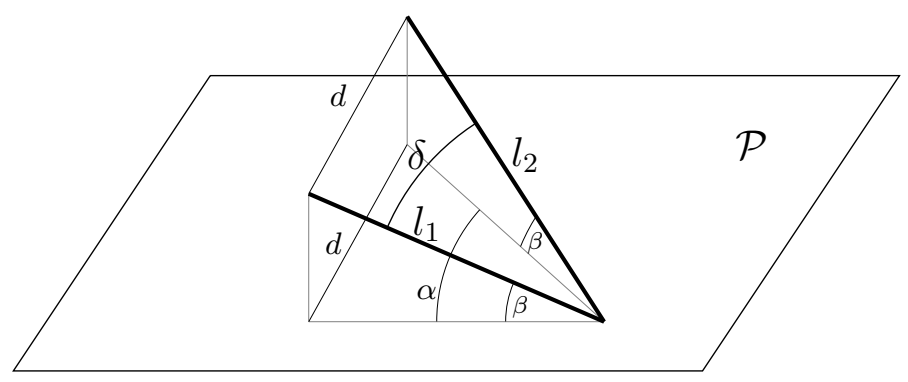

Fig. 3. Illustration of Lemma 1

For $\beta \leq \pi / 4$,

$$
\cos \delta \leq \cos \frac{\alpha}{2}
$$

which means that

$$
\delta \geq \frac{\alpha}{2}
$$

In addition, the following lemma will also be useful in our results.

Lemma 2. Consider two segments, $l_{1}$ and $l_{2}$, that share a common endpoint,

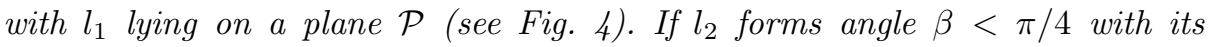
projection onto $\mathcal{P}$, then $\delta$, the angle between $l_{1}$ and $l_{2}$, is at least $\beta$.

Proof. Assume w.l.o.g. that $\left|l_{1}\right|=\left|l_{2}\right|=1$. Length of $a$, the projection of $l_{2}$ onto $\mathcal{P}$, is $\cos \beta$, and $h$, the distance of $l_{2}$ 's endpoint from $\mathcal{P}$ is $\sin \beta$. Let $\alpha$ be the angle between $l_{1}$ and $a$, and let $b$ be the segment connecting their endpoints. By the law of cosines,

$$
|b|^{2}=|a|^{2}+\left|l_{1}\right|^{2}-2|a|\left|l_{1}\right| \cos \alpha=\cos ^{2} \beta+1-2 \cos \beta \cos \alpha .
$$

Then,

$$
|d|^{2}=|h|^{2}+|b|^{2}=\sin ^{2} \beta+\cos ^{2} \beta+1-2 \cos \alpha \cos \beta=2(1-\cos \alpha \cos \beta) .
$$

Again, by the law of cosines,

$$
|d|^{2}=\left|l_{1}\right|^{2}+\left|l_{2}\right|^{2}-2\left|l_{1}\right|\left|l_{2}\right| \cos \delta=2(1-\cos \delta) .
$$

Comparing the two yields

$$
\cos \delta=\cos \alpha \cos \beta \text {. }
$$

Since $\cos \alpha \leq 1$, we get

$$
\cos \delta \leq \cos \beta
$$

and it follows that $\delta \geq \beta$. 


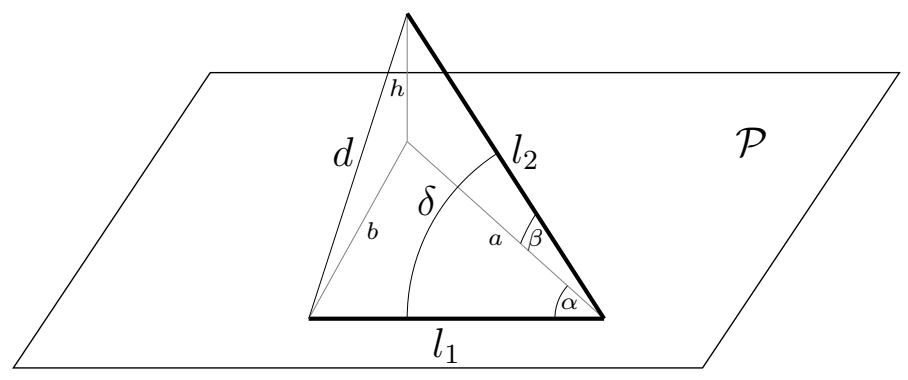

Fig. 4. Illustration of Lemma 2

\subsection{Vertices on a Sphere}

In this subsection, we consider the angular resolution obtained in a $3 \mathrm{D}$ arc diagram using straight-line edges drawn between vertices placed on a sphere. The two algorithms we present here are inspired by a two-dimensional drawing algorithm by Formann et al. 14. Our main result is the following.

Theorem 1. Let $G=(V, E)$ be a graph of degree $d$. There is a 3D straight-line drawing of $G$ with an angular resolution of $\Omega(1 / d)$, with the vertices of $G$ placed on the surface on a sphere.

Proof. Let $G^{2}=\left(V, E^{2}\right)$ be the square of $G$, that is the graph with the same set of vertices as $G$, and an edge between vertices $(u, v)$ if there is a path of length $\leq 2$ between $u$ and $v$ in $G$. Since $G$ has degree $d, G^{2}$ has degree $\leq d(d-1)<d^{2}$. Therefore, we can color the vertices of $G^{2}$ with at most $d^{2}$ colors, with the requirement that adjacent vertices have different colors.

We place the vertices on a unit sphere $\mathcal{S}$. We define $d^{2}$ cluster positions as follows. First, we cut the circle with $d+1$ uniformly spaced parallel planes (see Fig. (5), such that the maximum distance between the center of $\mathcal{S}$ and a plane is $h$ (thus, the distance between two neighboring planes is $2 h / d$ ). Then, we uniformly place $d$ points on each resulting circle. These are the cluster positions.

Since a coloring $\mathcal{C}$ of $G^{2}$ uses $\leq d^{2}$ colors, we can assign distinct cluster positions to colors in $\mathcal{C}$. To obtain a drawing of $G$, we place all vertices of the same color in $\mathcal{C}$ on the sphere, $\mathcal{S}$, within a small distance, $\epsilon$, around this color's cluster position, and draw edges in $E$ as straight lines. We can remove any intersections by perturbing the vertices slightly.

The claim is that the resulting drawing has resolution $\Omega(1 / d)$. Indeed, by setting $h=\pi /\left(\sqrt{1+\pi^{2}}\right)$, we get $\Omega(1 / d)$ minimal distance between any two planes, and $\Omega(1 / d)$ minimal distance between any two cluster positions on the same plane. So, the distance between any two cluster positions is at least $\Omega(1 / d)$.

Now let us consider any angle $\varangle a b c$ formed by edges $(a, b)$ and $(b, c)$. The edges forming $\varangle a b c$ define a plane, $\mathcal{P}$, whose intersection with $\mathcal{S}$ is a circle, $C$. Angle $\varangle a b c$ is inscribed in $C$, and based on the arc $\overparen{a c}$. Therefore, any other angle inscribed in $C$ and based on $\widehat{a c}$ has the same size, in particular the one formed 


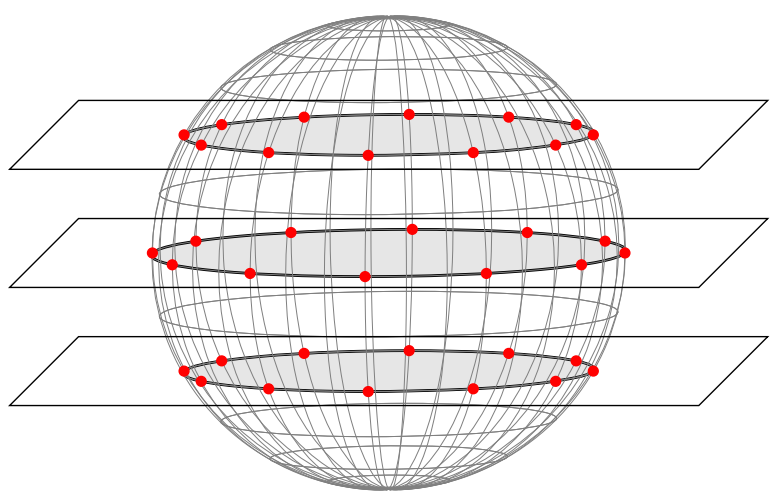

Fig. 5. Sphere cut with equidistant planes. Red points are the cluster positions.

by an isosceles triangle $\triangle a d c$. Since $|a d|=|c d| \leq 2(\mathcal{S}$ has radius 1$)$, and $|a c|$ is at least $\Omega(1 / d)$, then $|\varangle a b c|=|\varangle a d c|$ and is at least $\Omega(1 / d)$.

In addition, we also have the following.

Corollary 1. Let $G=(V, E)$ be a planar graph of degree $d$. There is a $3 D$ straight-line drawing of $G$ with an angular resolution of $\Omega\left(1 / d^{1 / 2}\right)$, with the vertices of $G$ placed on the surface of a sphere.

Proof. The proof is a direct consequence of applying the algorithm from the proof of Theorem 1 and the fact that the degree of $G^{2}$, the square of a planar graph, $G$, has degree $O(d)$ [14].

Thus, we can produce 3D arc diagram drawings of planar graphs that achieve an angular resolution that is within a constant factor of optimal. Admittedly, this type of drawing is probably not going to be very pretty when rendered, say, as a video fly-through on a $2 \mathrm{D}$ screen, as this type of drawing is unlikely to project to a planar drawing in any direction.

\subsection{Stationary Vertices}

In this subsection, we show how to overcome the drawback of the above method, in that we show how to start with any existing 2D straight-line drawing and dramatically improve the angular resolution for that drawing using a 3D arc diagram rendering that projects perpendicularly to the $2 \mathrm{D}$ drawing.

Theorem 2. Let $D(G)$ be a straight-line drawing of a graph, $G$, with arbitrary, but distinct, placements for its vertices in the base plane. There is a 3D arc diagram drawing of $G$ with the same vertex placements as $D(G)$ and with an angular resolution at least $\Omega(1 / d)$, where $d$ is the degree of $G$, regardless of the angular resolution of $D(G)$. 
Proof. Since we are not allowed to move vertices, and edges have to lie on planes perpendicular to the base plane, we are restricted to selecting angles $\alpha_{e}$ for edges $e$ of $G$. We do it by utilizing classical edge coloring, observing that the "entry" and "exit" angles for each vertex need to match.

First, we compute an edge coloring $\mathcal{C}$ of $G$ with $c$ colors $(c \leq d+1)$. Then, for each edge $e$, if its color in $\mathcal{C}$ is $i(i=0,1, \ldots, c-1)$, we set its angle to be $\alpha_{e}=i \cdot \pi / 4(c-1)$. For any two edges $e_{1}, e_{2}$, the difference between their angles $\alpha_{e_{1}}$ and $\alpha_{e_{2}}$ is at least $\pi / 4(c-1)$ (let $\alpha_{e_{1}}<\alpha_{e_{2}}$; consider the plane, $\mathcal{P}$, determined by both tangent lines having angle $\alpha_{e_{1}}$; the angle between $e_{2}$ and the plane $\mathcal{P}$, on which tangent of $e_{1}$ lies, is $\left.\alpha_{e_{2}}-\alpha_{e_{1}}\right)$. Therefore, by Lemma 2 the angle between $e_{1}$ and $e_{2}$ in the arc diagram is also at least $\pi / 4(c-1)=\Omega(1 / d)$.

It is unlikely that any pairs of the arcs touch each other in $3 \mathrm{D}$, but if any pair of them do touch, we can perturb one of them slightly to eliminate the crossing, while still keeping the angular separation for every pair of incident edges to be $\Omega(1 / d)$.

In addition, through the use of a slanted 3D arc diagram rendering, we can produce a drawing with angular resolution that is within a constant factor of optimal, with each arc projecting to its corresponding straight-line edge in some direction.

Theorem 3. Let $D(G)$ be a straight-line drawing of a graph, $G$, with arbitrary, but distinct, placements for its vertices in the base plane. There is a slanted $3 D$ arc diagram drawing of $G$ with the same vertex placements as $D(G)$ and with an angular resolution at least $\Omega\left(1 / d^{1 / 2}\right)$, where $d$ is the degree of $G$, regardless of the angular resolution of $D(G)$.

Proof. Let $C$ be a set of $\left\lceil d^{1 / 2}\right\rceil+1$ uniformly distributed angles from 0 to $\pi / 4$. Define a set of $d+1$ "colors" as distinct pairs, $(\alpha, \beta)$, where $\alpha$ and $\beta$ are each in $C$. Compute an edge coloring of $G$ using these colors. Now let $e$ be an edge in $G$, which is colored with $(\alpha, \beta)$. Draw the edge, $e$, using a circular arc that lies in a plane, $\mathcal{P}$, that makes an angle of $\alpha$ with the base plane and which has a tangent in $P$ that forms an angle of $\beta$ at each endpoint of $e$. (For instance, in Fig. 1 b, we give a slanted 3D arc diagram based on the edge coloring of the graph in Fig. 1 1a, corresponding to the following $\left(\alpha_{e}, \beta_{e}\right)$ "colors:" $\left(0^{\circ}, 0^{\circ}\right),\left(22.5^{\circ}, 0^{\circ}\right)$, $\left(45^{\circ}, 0^{\circ}\right),\left(22.5^{\circ}, 22.5^{\circ}\right),\left(45^{\circ}, 45^{\circ}\right)$.)

The claim is that every pair of incident edges is separated by an angle of size at least $\Omega\left(1 / d^{1 / 2}\right)$. So suppose $e$ and $f$ are two edges incident on the same vertex, $v$. Let $\left(\alpha_{e}, \beta_{e}\right)$ be the color of $e$ and let $\left(\alpha_{f}, \beta_{f}\right)$ be the color of $f$. Since $e$ and $f$ are incident and we computed a valid coloring for $G, \alpha_{e} \neq \alpha_{f}$ or $\beta_{e} \neq \beta_{f}$. In either case, this implies that $e$ and $f$ are separated by an angle of size at least $\Omega\left(1 / d^{1 / 2}\right)$ (by Lemma 1 if $\beta_{e}=\beta_{f}$, by Lemma 2 otherwise), which establishes the claim. As previously, we can perturb the arcs to eliminate crossings in 3D.

Thus, we can achieve optimal angular resolution in a 3D arc diagram for any graph, $G$, to within a constant factor, for any arbitrary placement of vertices of $G$ 
in the plane. Note, however, that even if $D(G)$ is planar, the $3 \mathrm{D}$ arc diagram this algorithm produces, when projected to the base plane, may create edge crossings in the projected drawing. It would be nice, therefore, to have $3 \mathrm{D}$ arc diagrams that could have good angular resolution and also have planar perpendicular projections in the base plane.

\subsection{Free Vertices}

In this section, we show how to take any $2 \mathrm{D}$ straight-line drawing with good angular resolution and convert it to a 3D arc diagram with angular resolution that is within a constant factor of optimal. Moreover, this is the result that makes use of a localized edge coloring.

Theorem 4. Let $D(G)$ be a straight-line drawing of a graph, $G$, with arbitrary, but distinct, placement for its vertices in the base plane, and $\Omega(1 / d)$ angular resolution. There is a $3 D$ arc diagram drawing of $G$ with the same vertex placements as $D(G)$ and with angular resolution at least $\Omega\left(1 / d^{1 / 2}\right)$, where $d$ is the degree of $G$, such that all arcs project perpendicularly as straight lines onto the base plane.

Proof. The algorithm is similar to the one from the proof of Theorem 2 , This time, however, we first compute an $L$-localized edge coloring, $\mathcal{C}$, of $G$ utilizing $c$ colors $(c \leq 2 L+1)$. Then, as previously, we assign angle $\alpha_{e}=i \cdot \pi / 4(c-1)$ to an edge $e$ of color $i$ in $\mathcal{C}(i=0,1, \ldots, c-1)$.

Let us consider two arcs, $e$ and $f$, incident on a vertex, $v$. If $\alpha_{e} \neq \alpha_{f}$, then the angle between $e$ and $f$ is at least $\pi /(4 c)=\Omega(1 / L)$, by Lemma 2 . Otherwise, $\alpha_{e}=\alpha_{f}$, and $e$ and $f$ have the same color in $\mathcal{C}$. By the definition of $L$-localized edge coloring, $e$ and $f$ are separated by at least $L / 2$ edges around $v$. Because $D(G)$ has resolution $\Omega(1 / d)$, the angle between $e$ and $f$ in $D(G)$ is $\Omega(L / d)$. Thus, by Lemma 1, the angle between $e$ and $f$ is also $\Omega(L / d)$. Therefore, the angle between $e$ and $f$ is $\Omega(\min \{1 / L, L / d\})$. We achieve the advertised angular resolution by setting $L=d^{1 / 2}$.

Theorem 4 shows that we can achieve $\Omega\left(1 / d^{1 / 2}\right)$ angular resolution in a $3 \mathrm{D}$ arc diagram drawing of a graph, $G$, with arcs projecting perpendicularly onto the base plane as straight-line segments, if there is a straight-line drawing of $G$ on a plane with an angular resolution of $\Omega(1 / d)$. The following is an immediate consequence.

Corollary 2. There is a $3 D$ arc diagram drawing of any planar graph, $G$, with straight-line projection onto the base plane, and an angular resolution of $\Omega\left(1 / d^{1 / 2}\right)$.

Proof. By [14, we can draw $G$ in a straight-line manner on a plane with an angular resolution of $\Omega(1 / d)$.

Admittedly, the 2D projection of this graph is not necessarily planar. We can nevertheless also achieve the following. 
Corollary 3. There is a $3 D$ arc diagram drawing of any ordered tree, $T$, with straight-line projection onto the base plane, and an angular resolution of $\Omega\left(1 / d^{1 / 2}\right)$.

Proof. By Duncan et al. [10, we can draw $T$ in a straight-line manner on a plane with an angular resolution of $\Omega(1 / d)$.

In addition, the area of the projection of the drawings produced by the previous two corollaries is polynomial.

\section{Conclusion}

We have given efficient algorithms for drawing 3D arc diagrams that achieve polynomial area in the base plane or sphere that contains all the vertices while also achieving good angular resolution. Since our algorithms deal with arc intersections via arc perturbation, the results may not be satisfactory, as the perturbed edges will still be very close. Therefore, one direction for future work is a related resolution question of what volumes are achievable if, in addition to angular resolution, we also insist that every circular arc always be at least unit distance from every other non-incident arc edge.

Acknowledgements. We thank Joe Simons, Michael Bannister, Lowell Trott, Will Devanny, and Roberto Tamassia for helpful discussions regarding angular resolution in $3 \mathrm{D}$ drawings.

\section{References}

1. Angelini, P., Eppstein, D., Frati, F., Kaufmann, M., Lazard, S., Mchedlidze, T., Teillaud, M., Wolff, A.: Universal point sets for planar graph drawings with circular $\operatorname{arcs}(2013)$ (manuscript)

2. Brandes, U.: Hunting down Graph B. In: Kratochvíl, J. (ed.) GD 1999. LNCS, vol. 1731, pp. 410-415. Springer, Heidelberg (1999)

3. Brandes, U., Shubina, G., Tamassia, R.: Improving angular resolution in visualizations of geographic networks. In: Leeuw, W., Liere, R. (eds.) Data Visualization, Eurographics, pp. 23-32. Springer (2000)

4. Brandes, U., Shubina, G., Tamassia, R.: Improving angular resolution in visualizations of geographic networks. In: Proc. Joint Eurographics — IEEE TCVG Symposium on Visualization (VisSym 2000) (2000)

5. Cheng, C., Duncan, C., Goodrich, M., Kobourov, S.: Drawing planar graphs with circular arcs. Discrete \& Computational Geometry 25(3), 405-418 (2001)

6. Cimikowski, A., Shope, P.: A neural-network algorithm for a graph layout problem. IEEE Trans. on Neural Networks 7(2), 341-345 (1996)

7. Cohen, R., Eades, P., Lin, T., Ruskey, F.: Three-dimensional graph drawing. Algorithmica 17(2), 199-208 (1997)

8. Djidjev, H.N., Vrt'o, I.: An improved lower bound for crossing numbers. In: Mutzel, P., Jünger, M., Leipert, S. (eds.) GD 2001. LNCS, vol. 2265, pp. 96-101. Springer, Heidelberg (2002) 
9. Duncan, C.A., Eppstein, D., Goodrich, M.T., Kobourov, S.G., Löffler, M.: Planar and poly-arc Lombardi drawings. In: Speckmann, B. (ed.) GD 2011. LNCS, vol. 7034, pp. 308-319. Springer, Heidelberg (2011)

10. Duncan, C.A., Eppstein, D., Goodrich, M.T., Kobourov, S.G., Nöllenburg, M.: Drawing trees with perfect angular resolution and polynomial area. In: Brandes, U., Cornelsen, S. (eds.) GD 2010. LNCS, vol. 6502, pp. 183-194. Springer, Heidelberg (2011)

11. Duncan, C.A., Eppstein, D., Goodrich, M.T., Kobourov, S.G., Nöllenburg, M.: Lombardi drawings of graphs. In: Brandes, U., Cornelsen, S. (eds.) GD 2010. LNCS, vol. 6502, pp. 195-207. Springer, Heidelberg (2011)

12. Eppstein, D., Löffler, M., Mumford, E., Nöllenburg, M.: Optimal 3d angular resolution for low-degree graphs. In: Brandes, U., Cornelsen, S. (eds.) GD 2010. LNCS, vol. 6502, pp. 208-219. Springer, Heidelberg (2011)

13. Fejes Tóth, L.: Über die Abschätzung des kürzesten Abstandes zweier Punkte eneis auf einer Kugelfläche liegenden Punktsystems. Jbf. Deutsch. Math. Verein 53, 66-68 (1943)

14. Formann, M., Hagerup, T., Haralambides, J., Kaufmann, M., Leighton, F.T., Symvonis, A., Welzl, E., Woeginger, G.J.: Drawing graphs in the plane with high resolution. In: FOCS, pp. 86-95. IEEE Computer Society (1990)

15. Garg, A., Tamassia, R.: Planar drawings and angular resolution: Algorithms and bounds. In: van Leeuwen, J. (ed.) ESA 1994. LNCS, vol. 855, pp. 12-23. Springer, Heidelberg (1994)

16. Garg, A., Tamassia, R., Vocca, P.: Drawing with colors. In: Díaz, J. (ed.) ESA 1996. LNCS, vol. 1136, pp. 12-26. Springer, Heidelberg (1996)

17. Goodrich, M.T., Wagner, C.G.: A framework for drawing planar graphs with curves and polylines. Journal of Algorithms 37(2), 399-421 (2000)

18. Gutwenger, C., Mutzel, P.: Planar polyline drawings with good angular resolution. In: Whitesides, S.H. (ed.) GD 1998. LNCS, vol. 1547, pp. 167-182. Springer, Heidelberg (1999)

19. Nicholson, T.: Permutation procedure for minimising the number of crossings in a network. Proc. of the Inst. of Electrical Engineers 115(1), 21-26 (1968)

20. Saaty, T.L.: The minimum number of intersections in complete graphs. Proceedings of the National Academy of Sciences of the United States of America 52(3), 688-690 (1964)

21. Tammes, P.: On the origin of number and arrangements of the places of exit on the surface of pollen grains. Rec. Trav. Bot. Neerl. 27, 1-81 (1930)

22. Vizing, V.G.: On an estimate of the chromatic class of a $p$-graph. Diskret. Analiz. 3, 25-30 (1964)

23. Wattenberg, M.: Arc diagrams: Visualizing structure in strings. In: IEEE Symp. on Information Visualization (InfoVis), pp. 110-116 (2002) 